\title{
Die Tabakprävention? Eine Schlüsselrolle für den Arzt
}

\author{
J. Cornuz, J.-P. Zellweger
}

La version française a paru dans le $n^{\circ} 47 / 2003$
Korrespondenz:

PD Dr. med. Jacques Cornuz, Département de médecine - IUMSP CHUV

CH-1011 Lausanne
Seit einigen Jahren wird die Tabakprävention zunehmend «medikalisiert». Das pharmazeutische Angebot erweitert sich fortlaufend und erlaubt es den Ärzten, eine diversifizierte Unterstützung für die Rauchenden anzubieten. Es gibt mittlerweile fünf Nikotinsubstitute (Depotpflaster, Kaugummi, Inhaler, Sublingualtablette und Lutschtablette) und seit kurzem ergänzt das Bupropion diese pharmazeutische Palette. Weitere Substanzen werden derzeit geprüft, wie das Rimonabant (Cannabinoidrezeptorblocker) und das Varenicline (Teilagonist an Nikotinrezeptoren). Zudem steht der Start von klinischen Versuchen mit der Nikotinimpfung kurz bevor!

Die internationalen (WHO) und nationalen Gesundheitsinstanzen (Bundesamt für Gesundheit) haben die Zeichen der Zeit verstanden. Die kürzliche Weltkonferenz «Tabak oder Gesundheit» in Helsinki hat die Hauptstossrichtungen des Kampfes gegen die Folgeschäden des Tabakkonsums erneut deutlich in Erinnerung gerufen. Diese Hauptachsen sind die Verschärfung der Gesetzgebung auf nationaler und internationaler Ebene, die Aufklärung der Öffentlichkeit, die Aus- und Fortbildung der Gesundheitsfachleute, insbesondere der Ärzte, die Unterstützung beim Rauchstopp durch geeignete Medikamente und schliesslich die Prävention bei Jugendlichen.

Diese Strategien werden durch die jüngste Rahmenkonvention der WHO zur Tabakkontrolle sowie das Nationale Programm zur Tabakprävention illustriert, Strategien, die auf eine kontinuierliche Reduktion der gesundheitlichen Tabakfolgeschäden zielen. Derzeit steht die Ausund Weiterbildung der Gesundheitsfachleute im Zentrum der Anstrengungen. Die meisten Länder bauen Weiterbildungsprogramme auf, welche die ärztlichen Kompetenzen in der wirksamen Rauchstoppberatung erhöhen wollen. Die Prävention, die bisher allzu häufig eher von Improvisation und gutem Willen abhing, basiert nun auf einer Reihe von klaren Daten und auf der Analyse jener Faktoren des Tabakkonsums, die den Einstieg wie auch den Ausstieg begünstigen.

Auch die Schweiz beteiligt sich an der allgemeinen Sensibilisierungs- und Weiterbildungsaktion für die Ärzteschaft. Das in der letzten Ausgabe der Schweizerischen Ärztezeitung enthaltene Angebot richtet sich an sämtliche Ärzte, die sich ihrer entscheidenden Rolle in der Tabakprävention bewusst sind, und bietet ihnen die Möglichkeit, ihre Kompetenzen zu verbessern. Das Fortbildungsangebot «Frei von Tabak» basiert auf einem überprüft wirksamen Programm [1] und wird von der Lungenliga Schweiz mit Unterstützung des Bundesamtes für Gesundheit (www.letitbe.ch) und weiterer Tabakpräventionsvereinigungen organisiert. Wir empfehlen den interessierten Kolleginnen und Kollegen, sich für diese Kurse anzumelden und sich aktiv zu beteiligen an der lohnendsten Präventionsaktion, die es im Bereich der öffentlichen Gesundheit überhaupt gibt.

\section{Literatur}

1 Cornuz J, Humair JP, Seematter L, Stoianov R, Van Melle G, Stalder H, Pécoud A. Efficacy of resident training in smoking cessation training: a randomized controlled trial of a program based on application of behavioral therapy and practice with standardized patients. Ann Intern Med 2002;136:429-37. 